\title{
Tracking and Reporting Outcomes in Medical Marijuana: Establishing Condition Specific State Level Registries
}

Paul C Langley, PhD

Adjunct Professor, College of Pharmacy, University of Minnesota

\begin{abstract}
In a previous commentary in INNOVATIONS in pharmacy, the question was raised as to the questions legislators should ask for the licensing of medical marijuana dispensaries. The case was made that if dispensaries accept they have a duty of care then they should be required to monitor patients over the course of their treatment with botanical cannabis, including hemp based product, to evaluate the response of patients to therapy. One option would be for individual dispensaries (or owners of multiple licenses and dispensary locations) to adopt a registry format and implement an on-line reporting system by registry staff and patients for the conditions being treated. Unfortunately, under present legislative rules for dispensaries there is no incentive for dispensaries to make the necessary investment. It is also unlikely that legislators would be prepared to mandate a registry requirement. The purpose of this commentary is to offer an alternative solution. Rather than dispensary specific registries, a state-wide low cost registry is proposed where dispensaries are required to log in and track patients with specific conditions. In the case of severe pain, a dispensary would log in patients presenting with this condition and the patient tracked over their course of treatment. A further advantage with a statewide registry is that if a patient visits a different dispensary they can still be tracked as they would be identified by their marijuana card number. The ability to track patients by condition, while still resident in a state, would not only minimize the issue of incomplete records, but would provide a comprehensive, research quality framework for evaluating claims for botanical cannabis. This could then provide feedback to legislators and establish a robust basis for rule making.
\end{abstract}

Keywords: Medical marijuana, outcomes assessment, state registry, rule making

\section{Introduction}

Do medical marijuana dispensaries have a duty of care to their patients? As noted in previous commentaries in INNOVATIONS in Pharmacy, if we accept the proposition that an approved medical marijuana dispensary is a healthcare provider, then it is under a reasonable obligation to ensure that the focus is on achieving the best possible clinical outcomes while at the same time endeavoring to minimize patient harms ${ }^{12} 3$. The notion of duty of care also implies that, in the delivery of care, the provider is obliged to attempt to ensure than the care is appropriate, that it is monitored and reviewed against targets set for the patient, and that the outcomes achieved are consistent with best clinical practice. Indeed, the case was made that a major oversight in approving the establishment of medical marijuana programs through commercially and notfor-profit operated dispensaries is the failure to put in place standards for the monitoring and reporting of outcomes. It was pointed out that the evidence base is limited for the range of dosing options, administrative routes and conditions treated.

Corresponding author: Paul C Langley, PhD

Adjunct Professor

College of Pharmacy University of Minnesota

Director, Maimon Research LLC

5061 North Apache Hills Trail, Tucson, AZ 85750

Tel: 520-577-0436; Email: langley@maimonresearch.com
The concern is that the ease that patients have in obtaining medical marijuana certification in many states means that a medical marijuana program may be, in effect, little different from a recreational program. Dispensaries understandably focus on sales and returns to investors with scant attention given to tracking and reporting outcomes across the range of conditions and symptoms presented. While this no doubt appeals to investors in reducing administration costs, it makes it virtually impossible to deliver the appropriate and coordinated level of care that patients should expect.

The solution proposed in one of the previous commentaries was to require, as part of the licensing process, that dispensaries meet certain reporting standards in respect of conditions treated. This, it was suggested, could be accomplished with dispensaries required to invest in a registry to track patients.

On reflection, there are a number of potential obstacles to a dispensary adopting a registry platform. Among these are: (i) it is unlikely state legislatures would make adoption of a registry format a condition of licensing; (ii) without a legislative requirement, there is no necessary incentive for dispensaries to invest in a registry; and (iii) as states allow patients to shop for their botanical cannabis product at different dispensaries, there is the risk of incomplete records. Indeed, even if a dispensary was prepared to invest in a registry, the risk is one of losing customers. Patients may see the reporting as burdensome and an invasion of privacy (even with HIPAA standards) and move 
their business to a 'registry-free' dispensary. In short, a registry model that is appropriate for a physician practice where patients have a longer term association and where there is the prospect of additional practice revenue through subscribing to a registry (as noted in previous commentaries for chronic pain and behavioral health), may not be as appropriate in botanical cannabis 45 .

Unfortunately, this still leaves the question of the limited evidence base for botanical cannabis and the failure of legislators to recognize that they are sanctioning botanical cannabis for conditions where the risk/benefit ratio is unknown. Indeed, recent large scale evaluations of the extent to which THC/CBD product characteristics offer symptom relief point to the lack of consensus on the risk/benefit ratios of THC versus CBD. In the recently reported study by Stith et al using mobile device software to evaluate real time effects, the authors concluded that across product characteristics, dried flower was the most commonly used and offered greater symptom relief that other types of products ${ }^{6}$. Further, among product characteristics 'only higher THC levels were independently associated with greater symptom relief and the presence of negative and positive side effects' while 'CBD potency levels were generally not associated with significant symptom changes or experienced side effects'. While the authors acknowledge the limitations, including selection bias, in their data and the lack of cross-referencing to clinical assessments (which the proposed registry structure overcomes), their results amplify the need for studies to address the impact of botanical cannabis in real world treating environments. As the authors conclude, there is a need for 'innovative strategies such as the use of mobile technology for measuring the multidimensional relationships between cannabis product characteristics, patient health conditions, perceived symptom relief, and side effect manifestation'.

\section{Dispensary and State Registries}

Ideally, there should be a mechanism in place that facilitates the adoption of a registry platform to evaluate the response to botanical cannabis. One approach, as noted above and in previous commentaries is for a dispensary owner to recognize not only that they have an implicit duty of care in evaluating outcomes of therapy but that, in what has become a highly competitive market in most states to acquire a dispensary license, the ability by applicants to differentiate themselves may resonate with state agencies. A commitment to a registry model may be seen, therefore, not only as a commitment to a duty of care but also recognition that it is important to improve the botanical cannabis evidence base. More to the point perhaps, committing to a registry would also show that the dispensary recognizes the need to provide feedback to providers who have recommended botanical cannabis as a therapy option. A registry which captures response to therapy utilizing validated outcomes instruments offers the opportunity to provide regular reports to providers so that a pain specialist, for example, can track response to therapy. A particular application here would be in assessing the risk/benefit ratio of botanical cannabis compared to opioids. The more comfortable pain specialists and primary care providers are with particular cannabis products and dosing regimens, the more willing they may be to consider, for example, botanical cannabis as a viable, evidence based adoption in the medium and long terms treatment of chronic pain. The provider, to extend the example, may require the patient to limit their choice of product to flowers with a known THC/CBD ratio, where the dispensary initiates therapy at a 'minimum' dose and on successive visits titrating the product to achieve a clinically meaningful response.

These arguments apply, of course, to more than just severe or chronic pain, which is the condition treated by $75-85 \%$ of patients at dispensaries. As detailed in the registry design described in a previous commentary the same arguments apply in the range of conditions typically treated (and mandated by state legislatures): persistent muscle spasms, severe nausea, post-traumatic stress disorder and seizures. In each case it is possible to track response to therapy through on-line patient self-reports, with product characteristic and dosing information supplied by the dispensary. A key point is the interaction between the provider and the dispensary to achieve and monitor a therapeutically meaningful response. If that is not achieved in an agreed timeframe then alternative interventions may be considered, to include polytherapy with a specific THC/CBD product.

The adoption of a state-level registry model would provide a complementary data source to individual dispensary registries. They key assumption here, of course, is how willing state authorities would be to facilitating a state-level registry model. The model proposed here would be one established as a standalone commercial product. It would sit alongside the established software systems in place for dispensaries to report to state health authorities and requirements for adverse event reporting and annual dispensary audits to maintain a license. Access would be through a web-based portal with reporting by both dispensary staff and patients. The structure would be little different from that proposed for the stand-alone dispensary registry. The key difference is the requirements for all dispensaries in the state to enroll patients who had specific medical conditions. At present, medical marijuana access, for those states with an integrated record system, is through conditions mandated by the legislature. A list that continues to expand through petitions from interested groups and associated bills passed by the legislature to approve access.

A state level registry would capture all conditions mandated by the legislature and provide a series of reports that patients completed specific to each mandated condition. In the case of severe or chronic pain, patients would report their status (i) in respect of overall pain status and (ii) pain by body location (e.g., thoracic, lumbar, knee) reporting through validated instruments pain intensity and functional status. Tracking 
patients by these validated clinical instruments from baseline (i.e., first dispensary visit) would give response to therapy with the response reported evaluated in terms of whether it is or is not clinically meaningful. Therapy response would also be assessed from a subjective assessment perspective using, as recommended in the previous commentary, the Patient Global Impression of Change (PGIC) instrument ${ }^{7}$. Adoption of the PGIC not only recognizes the importance of a global validated standard of response but a metric that can then be evaluated against validated instruments that assess clinical change specific to recognized conditions.

\section{Reporting Therapy Response}

From an evidence perspective, the critical issue is to be able to capture the product characteristics in terms of a standardized measure of THC and/or CBD. Two options are open: (i) to assess response by a cannabinoid THC/CBD using algorithms to create a standard measure or (ii) assess response by specific cannabinoid products. While these are not mutually exclusive (with the option of reporting on both) the rate limiting step is in the information available on the THC/CBD content of the various formulations of botanical cannabis: flower, oils and concentrates, topicals and edibles, together with the form of administration. One issue that legislators should consider is a standard label for these various products. Many cannabinoid products are mislabeled with little if any quality control of presumed 'same quality' products in successive production batches. It does not seem unreasonable that the manufacturing quality controls that are mandated for pharmaceutical products should not apply to cannabinoid products. It seems, therefore, surprising that legislators would agree to a new condition for botanical cannabis without specifying how the THC/CBD and form of administration is to be defined for reporting purposes. After all, the form of administration, even if there is agreement on (say) THC content may impact the effectiveness (if any) of the dosing (e.g., impact of sugar content of edibles). An 'open season' approach is unlikely to meet concerns of providers who may be considering botanical cannabis as a therapy option.

Assuming that legislation to support state registries, whether dispensary specific or a state-level condition specific registry, overcame the issue of a quality controlled, standard measure then we could consider reporting options. First, reporting to the individual dispensary would give useful feedback on the effectiveness of this registry in recommending (presumably) a specific product for specified conditions. From a duty of care perspective there may be concerns that different products (even with similar cannabinoid content) yield a range of therapeutic outcomes. Cannabinoid gummy bears may not have the therapeutic response of flowers. Questions may also be raised, not only against edibles (typically poorly labeled) but also for some 'marginal' products such as cannabinoid soap or bubble bath. Certainly, these may have topical effects (modified presumably by the duration of immersion in water) but it is also more in the realm of recreational rather than medical marijuana. Flower, it should be noted is the more typically consumed cannabinoid product (approximately $75 \%$ of patients) followed by concentrate. In fact, as noted by Stith et al, flower appears to provide more symptom relief relative to other products with pure indica sub-species providing more relief with sativa strains decreasing it. Again, the detail on comparing response by condition across product characteristics can set the groundwork for evidence-based guidelines relevant to dispensary operations, emphasizing that claims for relative efficacy of products need to be continually evaluated.

Second, both dispensary specific and state-level dispensaries should, as a matter of licensing in the former case, be required to submit detailed reports to the state health and legislative authorities on response to therapy. With registries in place this is low cost and straightforward as reporting can be automated.

Third, reporting to providers. If a provider signs off on a medical marijuana card application then, presumably, the provider is interested in the response to therapy? Unfortunately, in states such as Arizona, provider groups are established simply to exploit the demand for cards rather than being involved in any management plan for individual patients. They have no interest in long-term care or in tracking outcomes for patients. This leads the state open to the charge that it has in effect a de facto recreational marijuana program. As long as patients can meet the requisite assessment fees, then access to dispensaries is virtually guaranteed. It should be made clear by legislators that, under the duty of care umbrella, reports should be prepared and transmitted to the provider. Third-part intermediaries who are solely in the business of facilitating card applications should meet standards consistent with a duty of care. Under present administrative arrangements, such as Arizona, this is impossible unless the patient makes clear the card is issued with the knowledge and approval of the provider. All too often apparently, the provider is unaware that a medical card has been issued.

\section{Care Management}

As emphasized in previous commentaries, dispensary patients not only frequently present with more than one authorized condition (e.g., severe pain and severe nausea) but also can present with a range of other comorbidities. In behavioral health practices, for example, it has been suggested that some $30 \%$ of patients present with chronic pain associated with conditions such as depression and anxiety. If registries are to support a comprehensive data base then there has to be (i) the ability to track all conditions reported that are allowed by legislation and (ii) to record and, in some instances, capture highly prevalent comorbidities. Certainly, the registry should capture all conditions reported, together with a list of current medical conditions (coded appropriately to ICD-10-CMstandards). High prevalence co-morbidities, outside of conditions reported, would include: depression, fatigue, anxiety and sleep experience. 
It is important, in those states that have introduced recreational marijuana programs (following initiatives for medical marijuana) to separate these two markets. One way is to enforce rigorous standards for issuing medical marijuana cards (e.g., no intermediary provider groups whose sole business is initiating the card approval process where, not surprisingly, few fail to get approval and pay the requisite fee).

A second approach would be to encourage those bona fide card holders to stay within the medical marijuana market through price discrimination: applying higher sales taxes to products sold at the recreational marijuana counter (e.g., Colorado).

\section{Conclusions}

This commentary has presented two models for the monitoring and evaluation of treatment response for medical marijuana. The models presented are potentially complementary: (i) a state-level condition specific registry and (ii) a dispensary specific registry. Both models have their advantages and disadvantages. A state-level registry that mandated medical marijuana dispensary reporting for specific conditions may well run into legislative roadblocks and objections by interest groups. The dispensary model faces hurdles in the willingness of dispensaries to adopt a registry platform. This may be considered an unnecessary impost and one that may undercut their financial viability if patients switch to other dispensaries. Against this are arguments for a dispensary recognizing it has a duty of care; not only to track response to therapy but to establish links to providers as an active participant in the process of care. This may be driven by self-interest. Given the competition for dispensary licenses, a dispensary that has established links, offering an evidence-based approach to therapy management, may be in pole position for license applications and license renewals. After all, the dispensary model proposed here is attempting to alleviate the concerns of many state legislators that not only is the evidence base thin, but that medical marijuana claims are driven by interest groups aided by potential licensees. Establishing reporting requirements, which may be most attractive to those states that are yet to embrace a medical marijuana dispensary model, would not only alleviate concerns but also be a major step forward in establishing a robust evidence base.

Conflicts of Interest: None reported

\section{References}

${ }^{1}$ Langley PC. Outcomes, registries and medical marijuana: towards establishing dispensary monitoring and reporting standards. Inov Pharm. 2018; 9(4): Article 7

2 Langley PC. Establishing practice risk management and outcomes claims for medical marijuana dispensaries: Questions legislators should ask. Inov Pharm. 2019;10(1): Article 1

${ }^{3}$ Langley PC. Establishing credibility for medical marijuana: The proposed Prometheus Dispensary Registry for botanical cannabis. Inov Pharm. 2019;10(1):Article 2

${ }^{4}$ Langley PC. Meeting Physician Compliance Recommendations in the Management of Opioids in Chronic Pain: The Chronic Pain Management Registry (CPMR). Inov Pharm. 2019; 10(1): Article 13

${ }^{5}$ Langley PC. A Practice Based Behavioral Health Management Registry (BHMR): Implementation, Structure and Content. Inov Pharm. 2019. 10(2): Article 3

${ }^{6}$ Stith S, Vigil J, Brockleman F et al. The association between cannabis product characteristics and symptom relief. Scientific Reports. 2019;9:2712

${ }^{7}$ Hurst H, Bolton J. Assessing the clinical significance of change scores recorded on subjective outcome measures. $J$ Manipulative Physiol Ther. 2004;27:26-35 